\title{
The Knapsack Hash Function proposed at Crypto' 89 can be broken
}

\author{
Paul Camion Jacques Patarin
}

\begin{abstract}
INRIA, B.P. 105, Domaine de Voluceau - Rocquencourt, 78153 Le Chesnay Cedex France

EUROCRYPT'91, Brighton, England.
\end{abstract}

\begin{abstract}
Ivan Damgầrd [4] suggested at Crypto'89 concrete examples of hash functions among which a knapsack scheme. We will here show that a probabilistic algorithm can break this scheme with a number in the region of $2^{32}$ computations. That number of operations is feasible in realistic time with modern computers. Thus the proposed hash function is not very secure. Among those computations a substantial number can be performed once for all. A faster result can be obtained since parallelism is easy. Moreover, ways to extend the present algorithm to other knapsacks than the present $(256,128)$ suggested by Damgard are investigated.
\end{abstract}

\section{The proposed knapsack}

Let $a_{1}, \ldots, a_{s}$ be fixed integers of $A$ binary digits, randomly selected. If $T$ is a plaintext of $s$ binary symbols, $T=x_{1} \ldots x_{s}$, then $b=\sum_{i=1}^{s} x_{i} a_{i}$ will be the proposed hashed value. The values assigned are 256 for $s$ and 120 for $A$. Thus $b$ has at most $120+8=128$ binary digits. Thus roughly the probability that a random 256 - bit string be a solution is $2^{-128}$. We will see that it is somewhat larger. Here a probabilistic algorithm is however designed which solves the problem.

We nevertheless must emphasize that breaking that primitive (the proposed knapsack) does not allow us to break the hash function $h:\{0,1\}^{*} \rightarrow\{0,1\}^{t}$ whose construction is described in Theorem 3.1 of [4]. See also [7] for an improvement of that construction. We only show that the family $\mathcal{F}$ of functions defined by the proposed knapsack for $m=256$ and $t(m)=128$ is not collision free and hence does not satisfy the hypothesis of Theorem 3.1 of [4]. 


\section{The general scheme of our algorithm}

\subsection{Description}

$\underline{\text { Aim: }}$ Given $\mathrm{b}$, find a binary sequence $x_{1}, x_{2}, \ldots, x_{s}$ such that $\sum_{i=1}^{256} x_{i} a_{i}=b$.

This is a Knapsack problem, which has an expected high number of solutions, i.e at first sight $\simeq \frac{2^{256}}{2^{128}}$ and we will show a way to find one of those solutions.

Step 0 : We choose integers $m, m_{1}, m_{2}, m_{3}, m_{4}$ such that

a) $m=m_{1} m_{2} m_{3} m_{4}>2^{128}$

b) The $m_{i}$ are pairwise coprime, and $m_{i} \simeq 2^{32}, i=1,2,3,4$.

Let $b_{i}$ be the residue of $b$ modulo $m_{i}, i=1,2,3,4$. By the chinese remainder theorem we have that $x=b$ is the only integer such that

1. $x \equiv b_{i}\left[m_{i}\right], i=1,2,3,4$.

2. $0 \leq x<m$.

Here is a diagram of the sequence of operations that is considered. Let us sketch the meaning of the diagram before going into details.

Each black point represents a step resulting in an estimated $2^{32}$ binary sequences. The length of those sequences is 64 for steps $1,2,3,4$. It is 128 for steps 5 and 6 and finally step 7 produces about $2^{32}$ sequences of length 256 among which test modulo $m_{4}$ selects a solution with probability close to 1 .

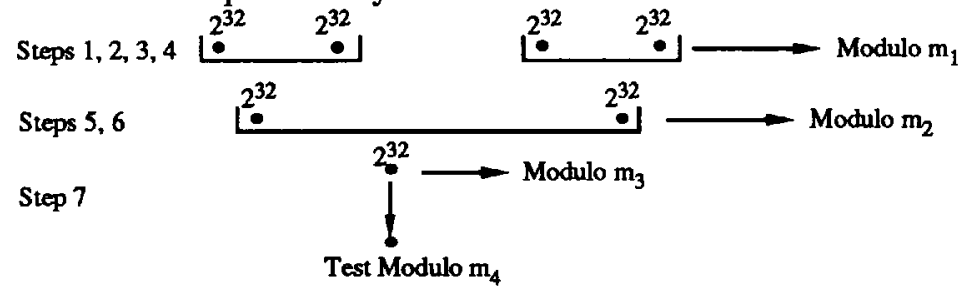

We now go through each step in detail.

Step 1 : We find all sequences $\left(x_{i}\right), 1 \leq i \leq 64, x_{i}=0$ or 1 , such that

$$
\sum_{i=1}^{64} x_{i} a_{i} \equiv b_{1}\left[m_{1}\right]
$$

We will find about $2^{32}$ such sequences because there are $2^{64}$ sequences $\left(x_{i}\right)$ of 64 bits, and $m_{1}$ is close to $2^{32}$. In fact, we will examine in Section 2 what is the expected number of solutions to be obtained when the algorithm is brought to completion. 
Important remark. Finding sequences $\left(x_{i}\right)$ such that $\sum_{i=1}^{64} x_{i} a_{i} \equiv b_{1}\left[m_{1}\right]$ needs a number in the region of $2^{32}$ operations, if a memory with sufficient size is available.

Indeed, we just have to do the following.

a) compute and store all values of $b_{1}-\sum_{i=1}^{32} x_{i} a_{i}$ modulo $m_{1}$.

b) compute and store all values of $\sum_{i=33}^{64} x_{i} a_{i}$ modulo $m_{1}$.

c) keep all pairs of sequences $\left(x_{i}\right)$ which give the same value modulo $m_{1}$ in a) and b).

More details. In Section 3 we give more details about operations a), b), c), about the memory needed and about the number of solutions to be found.

Step 2 : The same way, we find about $2^{32}$ binary sequences $\left(x_{i}\right)$ such that $\sum_{i=65}^{128} x_{i} a_{i} \equiv$ $0\left[m_{1}\right]$.

Step 3 : The same way, we find about $2^{32}$ binary sequences $\left(x_{i}\right)$ such that $\sum_{i=129}^{192} x_{i} a_{i} \equiv$ $0\left[m_{1}\right]$.

Step 4 : The same way, we find about $2^{32}$ binary sequences $\left(x_{i}\right)$ such that $\sum_{i=193}^{256} x_{i} a_{i} \equiv$ $0\left[m_{1}\right]$.

Step 5 : We denote $\sum_{i=1}^{64} x_{i} a_{i}$ by $s_{1}$ and $\sum_{i=65}^{128} x_{i} a_{i}$ by $s_{2}$.

From the sequences $\left(x_{i}\right)$ found at Steps 1 and 2, and using the procedure a) b) c) above, we find about $2^{32}$ sequences $\left(x_{i}\right), 1 \leq i \leq 128$ such that $s_{1}+s_{2} \equiv b_{2}\left[m_{2}\right]$. For there are about $2^{32} \times 2^{32}=2^{64}$ sequences $\left(x_{i}\right), 1 \leq i \leq 128$ such that $\left(x_{1}, \ldots, x_{64}\right)$ is a solution in Step 1 and $\left(x_{65}, \ldots, x_{128}\right)$ is a solution in Step 2. So if the numbers $s_{1}+s_{2}$ are about equally distributed modulo $m_{2}, m_{2} \simeq 2^{32}$, we find about $\frac{2^{64}}{2^{32}}=2^{32}$ among those sequences such that $s_{1}+s_{2} \equiv b_{2}\left[m_{2}\right]$. If we find noticeably less than $2^{32}$ such solutions, we will see at the end of this Section 1 what to do.

All sequences $\left(x_{i}\right)$ to be found in Step 5 also have the following property:

$$
s_{1}+s_{2} \equiv b_{2}\left[m_{2}\right] \text { and } s_{1}+s_{2} \equiv b_{1}\left[m_{1}\right] \text {. }
$$

This is because $s_{1} \equiv b_{1}\left[m_{1}\right]$ and $s_{2} \equiv 0\left[m_{1}\right]$.

Remark Step 5 also uses a number in the region of $2^{32}$ operations. The computer problem is the same as for step 1 . Details will be given in Section 3. We essentially have two sets $E$ and $F$ of about $2^{32}$ elements, and we want to find all possible couples $(a, b), a \in E$, $b \in F$, such that $a=b \equiv k\left[m_{i}\right]$, where $k$ is a fixed value and $m_{i}$ is close to $2^{32}$. 
Step 6 : The same way, from the sequences $\left(x_{i}\right)$ found at Steps 3 and 4 , we find about $2^{32}$ sequences $\left(x_{i}\right), 129 \leq i \leq 256$, such that

if $s_{3}=\sum_{i=129}^{192} x_{i} a_{i}$ and $s_{4}=\sum_{i=193}^{256} x_{i} a_{i}$, we have that $s_{3}+s_{4} \equiv 0\left[m_{2}\right]$.

Moreover $s_{3}+s_{4} \equiv 0\left[m_{1}\right]$, since $s_{3} \equiv 0\left[m_{1}\right]$ and $s_{4} \equiv 0\left[m_{1}\right]$.

Step 7 : The same way, but now from the sequences $\left(x_{i}\right)$ found at the Steps 5 and 6 , we find about $2^{32}$ sequences $\left(x_{i}\right), 1 \leq i \leq 256$ such that $\left(s_{1}+s_{2}\right)+\left(s_{3}+s_{4}\right) \equiv b_{3}\left[m_{3}\right]$.

By construction, we also have that

$$
s_{1}+s_{2}+s_{3}+s_{4} \equiv b_{1}\left[m_{1}\right] \text { and } s_{1}+s_{2}+s_{3}+s_{4} \equiv b_{2}\left[m_{2}\right] .
$$

Modulo $m_{4}$, we have about $2^{32}$ possible values, indeed $m_{4} \simeq 2^{32}$. Since we found $2^{32}$ sequences $\left(x_{i}\right)$, there is a high probability that at least one of these sequences is such that $s_{1}+s_{2}+s_{3}+s_{4} \equiv b_{4}\left[m_{4}\right]$. We will see in section 2 that we generally find more than one such sequence. We will see at the end of this section what to do if we don't find any such sequence.

Now, suppose that we have such a sequence.

$$
s_{1}+s_{2}+s_{3}+s_{4}=\sum_{j=1}^{256} x_{j} a_{j} .
$$

That sequence $\left(x_{i}\right)$ is such that

1. $\sum_{j=1}^{256} x_{j} a_{j} \equiv b_{i}\left[m_{i}\right], i=1,2,3,4$.

2. $0 \leq \sum_{j=1}^{256} x_{j} a_{j} \leq 256 \cdot 2^{120}=2^{128}$.

Thus by a) and b) in Step 0 , we finally found a sequence $\left(x_{i}\right)$ such that $\sum_{j=1}^{256} x_{j} a_{j}=b$, as desired.

A careful justification of the fact that an average number of $2^{32}$ solutions are to be found in step 7 is a consequence of corollary 1 in section 2.3 . It essentially relies on the fact that the sum of two mutually independent uniformly distributed random variables (m.i.u.d.r.v.) over an abelian group is itself a m.i.u.d.r.v..

Remark What are we going to do if at any a step we have got much less than $2^{32}$ solutions, or if at the end we don't find any solution such that $s_{1}+s_{2}+s_{3}+s_{4} \equiv b_{4}\left[m_{i}\right], i=1,2,3,4$ ?

Then it is possible to use the algorithm again, but with new chosen values. For example we can replace $b_{1}$ by $s_{1} \equiv b_{1}-\lambda\left[m_{1}\right]$ at Step 1 , and 0 by $s_{2} \equiv \lambda\left[m_{1}\right]$ at Step 2, where $\lambda$ is any fixed integer in $\left[0, m_{1}-1\right]$.

Or we can even just permute the $a_{i}$ 's.

At each try, we will have a high probability of success, so the probability of success after a few tries can be as close to 1 as desired. 


\section{Proof}

\subsection{The collision free functions family}

In this section the given sequence element from $\left[0,2^{120}\left[{ }^{n}\right.\right.$, is denoted by $a^{*}=\left\{a_{1}^{*}, a_{2}^{*}, \ldots, a_{n}^{*}\right\}$ and the value for which a collision is searched is denoted by $b^{*}$.

Let us recall what we need form the definition in [5] of a collision free function family $\mathcal{F}$. Such a family is given by an infinite family of finite sets $\left\{F_{n}\right\}_{n=1}^{\infty}$, and a function $t: \mathbf{N} \rightarrow \mathbf{N}$, such that $t(n)<n$ for all $n \in \mathbf{N}$.

Now a member of $F_{n}$ here is a function

$$
f_{a}:\{0,1\}^{n} \rightarrow\{0,1\}^{t}
$$

defined by

$$
x \sim \sum_{i=1}^{n} x_{i} a_{i}
$$

where $a=\left(a_{1}, \ldots, a_{n}\right)$ belongs to the product $\mathcal{A}=\left[0,2^{\ell}\left[{ }^{n}\right.\right.$ of $n$ intervals of integers, for $\ell=t-\log n$.

Integer $\sum_{i=1}^{n} x_{i} a_{i}$ is considered a binary sequence which is its writing in the radix two. Function $f_{a}$ is called an instance of $\mathcal{F}$ of size $n$.

We here consider the set $F_{n}$ for which $n=256$ and $t(n)=128$. However the argument here does not generally depend on specifie values of $n$ or other parameters but if so we will point it out.

\subsection{Some questions to be replied}

The particular function $f_{a}$ that will be analyzed is given by an $a$ randomly selected from $\mathcal{A}$.

The set $\mathcal{A}$ is thus made a sample space.

For this specific application it has the equally likely $r$ obability measure but our argument can be exploited in more general situations.

To clarify, let us state one of the problems we will be faced with. Let $q$ be an integer ( $q$ is large but small compared to $2^{\ell}$ ).

Given $b$ in $\left[0,2^{t}[\right.$, the following expectation will be estimated

$$
e(b)=\sum_{a \in \mathcal{A}} p(a)\left|\left\{x \mid x \in\{0,1\}^{n}, f_{a}(x) \equiv b[q]\right\}\right|
$$

where $p$ is the probability measure on $\mathcal{A}$.

Other expectations are needed as the following

$$
e_{j}(b)=\sum_{a \in \mathcal{A}} p(a) \mid\left\{x \mid x \in\{0,1\}^{n}, f_{a}(x) \equiv b[q] \text { and }|s(x)|=j\right\} \mid, j=0, \ldots, n,
$$


where the support $s(x)$ of $x$ is defined as

$$
s(x)=\left\{i \mid x_{i}=1\right\} .
$$

We will in particular observe that those expectations don't depend on a particular choice of a non zero $b$, for a non zero $j$.

\subsection{Random variables}

The boolean function $\phi$ (statement) takes the value 1 if "statement" is true and 0 otherwise. We here introduce random variables (briefly r.v.) defined on $\mathcal{A}$ and with values in an abelian group.

Let $q$ be an integer relatively small compared to $2^{\ell}$. The additive group $(\mathbf{Z} / q \mathbf{Z},+)$ of integers modulo $q$ is the abelian group denoted by $G_{q}$. Let $I=\left[0,2^{\ell}[\right.$ be a sample space with the equally likely probability measure.

Then the mapping

$$
\theta: I \rightarrow G_{q}
$$

defines a r.v. which may be considered uniformly distributed since $q$ is negligeable compared to $\frac{2^{\ell}}{q}$.

Moreover we will consider sums of mutually independent (briefly m.i.) r.v. identical to $\theta$ and it is not difficult to prove that for whatever probability distribution, (briefly p.d.) at the only condition that the considered random variable with values in an abelian group has a positive probability on each element of a set of generators of that group, then the p.d. of the sum of a large number of m.i.r.v. identical to the given one approaches a uniform distribution.

To every subset $J$ of $[1, n]$ corresponds a random variable $\theta_{J}: \mathcal{A} \rightarrow G_{q}$ defined by

$$
\forall a \in \mathcal{A}: \theta_{J}(a)=\sum_{j \in J} a_{j} \in G_{q}
$$

and we thus have

$$
P_{r}\left\{\theta_{J}=b\right\}=\sum_{a \in \mathcal{A}, \theta_{J}(a)=b} P(a) .
$$

For convenience we also define $\theta_{\emptyset}$ by

$$
\forall a \in \mathcal{A}: \theta_{\mathbb{\vartheta}}(a)=0 \in G_{q}
$$

Clearly $\theta_{J}$ is a sum of $|J|$ m.i.r.v. identical to $\theta$ and $\theta_{J_{1}}=\theta_{J_{2}}$ for $\left|J_{1}\right|=\left|J_{2}\right|$.

We thus denote by $\theta_{j}$ the common r.v. $\theta_{J}$ with $|J|=j$ and $\theta_{\emptyset}$ is denoted by $\theta_{0}$.

We then have that

$$
P_{r}\left\{\theta_{\emptyset}=b\right\}=\phi(b=0) .
$$

We now classically have the

Theorem 1 Given $b$, then the expectation $e(b)$ is worth

$$
\sum_{a \in \mathcal{A}} p(a)\left|\left\{x \mid x \in\{0,1\}^{n}, f_{a}(x) \equiv b[q]\right\}\right|=\sum_{x \in\{0,1\}^{n}} P_{r}\left\{\theta_{s(x)}=b\right\} .
$$




\section{Proof}

The L.H.S. is worth

$$
\begin{aligned}
& \sum_{(a, x) \in \mathcal{A} \times\{0,1\}^{n}} p(a) \phi\left(\sum_{i=1}^{n} x_{i} a_{i} \equiv b[q]\right) \\
= & \sum_{x \in\{0,1\}^{n}} \sum_{a \in \mathcal{A}, \sum_{i=1}^{n} x_{i} a_{i} \equiv b[q]} p(a),
\end{aligned}
$$

which is nothing but the R.H.S.

Corollary 1 If $\theta$ is a uniformly distributed random variable (briefly u.d.r.v.) then the expectation considered in Theorem 1 is worth

$$
\left(2^{n}-1\right) / q+\phi(b=0) .
$$

Corollary 2 Given an integer $j, 0 \leq j \leq m$, the expectation

$$
e_{j}(b)=\sum_{a \in \mathcal{A}} p(a) \mid\left\{x \mid x \in\{0,1\}^{n}, f_{a}(x) \equiv b[q] \quad \text { and }|s(x)|=j\right\}
$$

is worth

$$
\left(\begin{array}{c}
n \\
j
\end{array}\right) P_{r}\left\{\theta_{j}=b\right\}
$$

If $\theta$ is a u.d.r.v., then this is $\left(\begin{array}{c}n \\ j\end{array}\right) / q$ for $j>0$ and $\phi(b=0)$ for $j=0$.

\subsection{The expected weight distribution in the sample to be scanned}

\subsubsection{The expected number of collisions}

Let $m_{1}, m_{2}, m_{3}, m_{4}$ be the integers given in section 1 and take $q=m_{1} m_{2} m_{3}$.

Since $q \simeq 2^{96}<b^{*}$ then the set

$$
R=\left\{x \in\{0,1\}^{n}, f_{x^{*}}(x) \equiv b^{*}[q]\right\}
$$

contains the set $M$ of all $x$ in $\{0,1\}$ such that $f_{a^{\prime}}(x)=b^{*}$ from which we have to exhibit a member, among other $0-1$ sequences. By Theorem 1 we can assess that the expected size $e\left(b^{*}\right)$ of $R$ is $2^{n} / q=2^{160}$.

By Corollary 2 , the weight ratio distribution in $R$ is the same as is $\{0,1\}^{n}$ i.e.

$$
\left(\frac{\left(\begin{array}{c}
n \\
j
\end{array}\right)}{2^{n}}\right)_{j=0}^{n}
$$


The size of $M$ may be in its turn assessed.

The set $\{0,1\}^{n}$ being assumed to have the equally likely probability measure we have that

$$
\operatorname{Pr}\left\{n p-t \leq \sum_{i=1}^{n} x_{i} \leq n p+t\right\}=\sum_{n p-t \leq i \leq n p+t}\left(\begin{array}{c}
n \\
i
\end{array}\right) p^{i} q^{n-i}
$$

For $n=256, p=q=\frac{1}{2}$ and $t=20$, this is worth 0.9897 . This means that in most cases, $b$ will lie in the interval $\left[108.2^{119}, 148.2^{119}\right]$, so that there are about $41.2^{119} \simeq 2^{124.35}$ possible values for $b$. Thus when a value $b^{*}$ is assigned to $b$ in that range then the size of $M$ is in the region of $2^{256-124.35}=2^{131.64}$.

Now $M$ is contained in $R$.

This means that the probability to obtain a collision when drawing an element from $R$ at random is $2^{131.64-160}=2^{-28.35}$.

If we could operate such drawings about $2^{32}$ times, then the expected number of collisions obtained would be $2^{-28.35} \times 2^{32}=2^{3.64}>12$.

Actually our algorithm consists in constructing a subset $S$ of $R$ the expected size of which is $2^{32}$ in which the expected number of collisions is still $2^{3.64}$.

\subsubsection{A suitable partition of the set $R$}

Given a partition $E_{1} \cup E_{2} \cup E_{3} \cup E_{4}$ of $[1, n]$ with $\left|E_{i}\right|=n / 4, i=1, \ldots, 4$ we define a partition $\left\{S_{\gamma}\right\}_{\gamma \in \Gamma}$ of $R$, where $\Gamma=G_{m_{1}} \times G_{m_{1}} \times G_{m_{1}} \times G_{m_{2}}, \gamma$ being a quadruple $\left(c_{1}, c_{2}, c_{3}, d_{1}\right)$.

The set $S_{\gamma}$ is defined as

$$
\left\{x \mid x \in R, \sum_{i \in E_{j}} x_{i} a_{i}^{*} \equiv c_{j}\left[m_{1}\right], j=1,2,3, \sum_{i \in F_{1}} x_{i} a_{i}^{*} \equiv d_{1}\left[m_{2}\right]\right\}
$$

where $F_{1}=E_{1} \cup E_{2}$. Also $F_{2}=E_{3} \cup E_{4}$.

Notice that for $x$ in $S_{\gamma}$, since $S_{\gamma} \subset R$, we necessarily have that $\sum_{i \in E_{4}} x_{i} a_{i}^{*} \equiv b^{*}-c_{1}-c_{2}-c_{3}\left[m_{1}\right], \sum_{i \in F_{2}} x_{i} a_{i}^{*} \equiv b^{*}-d_{1}\left[m_{2}\right]$ and $\sum_{i=1}^{n} x_{i} a_{i}^{*} \equiv b^{*}\left[m_{3}\right]$. Clearly by the general property of $\theta$ defined in (2.3) the conditional probability

$$
P_{r}\left\{x \in S_{\gamma} \mid x \in R\right\}
$$

is very close to $1 / m_{1}^{3} m_{2}$ and thus Theorem 1 entails that the expected size of $S_{\gamma}$ is $|R| / m_{1}^{3} m_{2}$ which in the example dealt with is $2^{32}$.

Notice that in the description of the algorithm, $c_{1}$ was chosen to be equal to $b^{*} \bmod m$, and $c_{2} \equiv c_{3} \equiv 0[m]$, also $d_{1} \equiv b^{*}\left[m_{2}\right]$. We next give evidence that the expected size of $S_{\gamma} \cap M$ does not depend on a particular choice of $\gamma$.

We will need a corollary to corollary 2 of Theorem 1. 
Corollary 3 For any $\gamma \in \Gamma$, the weight ratio distribution in $S_{\gamma}$ is very close to

$$
\left(\frac{\left(\begin{array}{c}
n \\
j
\end{array}\right)}{2^{n}}\right)_{j=0}^{n}
$$

Indeed for every $J$ for which $J \cap E_{i} \neq \emptyset, i=1,2,3,4$, we have that

$$
\begin{aligned}
& P_{r}\left(x \in S_{\gamma} \text { and } s(x)=J\right) \\
& =\prod_{j=1}^{4} P_{r}\left(\sum_{i \in J \cap E_{j}} x_{i} a_{i} \equiv c_{j}\left[m_{1}\right]\right) \prod_{j=1}^{2}\left(\sum_{i \in J \cap F_{j}} x_{i} a_{i} \equiv d_{j}\left[m_{2}\right]\right) P_{r}\left(\sum_{i=1}^{n} x_{i} a_{i} \equiv b\left[m_{3}\right]\right)
\end{aligned}
$$

$=1 / m_{1}^{4} m_{2}^{2} m_{3}$, where $\gamma=\left(c_{1}, c_{2}, c_{3}, d_{1}\right)$ and $c_{4} \equiv b-c_{1}-c_{2}-c_{3}\left[m_{1}\right], d_{2} \equiv b-d\left[m_{2}\right]$. Moreover the contribution of the sets $J$, such that $J \cap E_{i}=\emptyset$ for some $i$, to the expectation

$$
\sum_{a \in \mathcal{A}} p(a)\left|\left\{x\left|x \in S_{\gamma},\right| s(x) \mid=j\right\}\right|
$$

being negligeable tor the relevant sizes of $j$, then a similar argument as the one used for proving corollary 2 proves the thesis.

\subsection{Sums of random variables with integer values}

The expected size of $M$, given $b$, is

$$
\sum_{a \in \mathcal{A}} P(a)\left|\left\{x \mid x \in\{0,1\}^{n}, \sum_{i=1}^{n} x_{i} a_{i}=b\right\}\right| .
$$

A random variable $\omega_{J}: a \sim \sum_{i \in J} a_{i}$ mapping $\mathcal{A}$ into $\left[0,2^{t}[\right.$ corresponds to each subset $J$ of $[1, n]$. Notice that $\operatorname{Pr}\left\{\omega_{J}=b\right\}$ here depends on $b$ and $J$ when $\mathcal{A}$ has the equally likely probability measure. But by symmetry, for any $b, P_{r}\left\{\omega_{J}=b\right\}$ only depends on the size of $J$.

By the same argument as for Theorem 1 we have that the expected size of $M$ is

$$
\sum_{J \subset[1, n]} P_{r}\left\{\omega_{J}=b\right\}
$$

We now consider any partition $H_{1} \cup H_{2}=[1, n]$. To obtain the evidence claimed in section 2.4.2, we need to show that given $q$ small and any $J \subset[1, n]$ such that $J \cap H_{1} \neq \emptyset$, we have that

$$
P_{r}\left\{\sum_{i \in H_{1} \cap J} x_{i} a_{i} \equiv c[q] \mid x \in M\right\}
$$

very slightly depend on the choice of $c$. Thus the expected size of $M \cap S_{\gamma}$ will only slightly depend on the choice of $\gamma$.

We have that

$$
\omega_{J}(a)=\sum_{i \in J \cap H_{1}} a_{i}+\sum_{i \in J \cap H_{2}} a_{i}
$$


and for $\omega_{J}(a)=b$, then if $J \cap H_{1}$ is not empty, $\sum_{i \in J \cap H_{1}} a_{i}$ takes all integer values from 0 to $b$, as a runs over $\mathcal{A}$. We will observe on some numerical values that over $q$ successive values $v_{1}, \ldots, v_{1}+q-1$ taken by $v$ in $[0, b]$ then the variation of $P_{r}\left\{\omega_{J \cap H_{1}}=v\right\}$ is negligeable. Now in view of corollary 3 , for a given partition $H_{1} \cup H_{2}=[1, n]$ where the smallest size of $H_{i}$ is $64, i=1,2$ then the contribution to

$$
P_{\tau}\left\{\sum_{i \in H_{1}} x_{i} a_{i} \equiv c[q] \mid x \in M\right\}
$$

of $x$ 's such that $s(x) \cap H_{1}=\emptyset$ is negligeable since most of the weights of $x$ 's in $S_{\gamma}$ are in the interval $[118,148]$, by corollary 3 , and

$$
\left(\begin{array}{c}
256-64 \\
118
\end{array}\right) /\left(\begin{array}{l}
256 \\
118
\end{array}\right)=8.6510^{-22} .
$$

We use the following result on the probability distribution of the sum of $j$ m.i.u.d.r.v. with values in the interval of integers $[0, h[$.

([4] page 52).

Let $c$ be in $\left[0, j h\left[\right.\right.$. As $c$ and $h$ grow indefinitely, their ratio $\frac{c}{h}$ approaching $\xi$ which is a real number in $[0, j[$, then the probability distribution

$$
a_{j, c}=P_{r}\left\{X_{1}+X_{2}+\ldots+X_{j}<c\right\}
$$

tends to

$$
F(\xi)=\lim _{\frac{c}{h} \rightarrow \xi} a_{j, c}=\frac{1}{j !} \sum_{\nu<\xi}\left(\begin{array}{l}
j \\
\nu
\end{array}\right)(\xi-\nu)^{j} .
$$

We compute $F(\xi)$ and $F(\xi+\varepsilon)$ for $\varepsilon=\frac{q}{h}=\frac{2^{32}}{2^{120}}=2^{-88}$ and for a few values of $j$ and $x$. Since $F(\xi)$ is increasing we only have to notice that $F(\xi+\varepsilon)$ is very close to $F(\xi)$ to be convinced of the claim in 2.4.2. We also compute some values for $\varepsilon=2^{-10}$ to show that for $q$ in the region of $2^{110}$ we would move away from our assumption.

\begin{tabular}{|c|c|c|c|c|c|}
\hline$j$ & $\xi$ & $\varepsilon$ & $F(\xi)$ & $F(\xi+\varepsilon)-F(\xi)$ & Ratio \\
\hline 32 & 15 & $2^{-88}$ & $2.710^{-1}$ & $6.5310^{-28}$ & $2.4110^{-27}$ \\
\hline 32 & 5 & $2^{-88}$ & $8.6210^{-14}$ & $1.7710^{-39}$ & $2.0510^{-26}$ \\
\hline 15 & 7 & $2^{-88}$ & $3.2810^{-1}$ & $1.0410^{-27}$ & $3.1510^{-27}$ \\
\hline 15 & 3 & $2^{-88}$ & $1.0610^{-5}$ & $1.6810^{-31}$ & $1.5910^{-26}$ \\
\hline \hline 32 & 15 & $2^{-10}$ & $2.7110^{-1}$ & $1.9710^{-4}$ & $7.2910^{-4}$ \\
\hline 32 & 5 & $2^{-10}$ & $8.6210^{-14}$ & $2.1710^{-15}$ & $2.5110^{-2}$ \\
\hline 15 & 7 & $2^{-10}$ & $3.2910^{-1}$ & $3.1310^{-4}$ & $9.5310^{-4}$ \\
\hline 15 & 3 & $2^{-10}$ & $1.0610^{-5}$ & $5.0910^{-8}$ & $4.810^{-3}$ \\
\hline
\end{tabular}




\section{More details about the algorithm}

\subsection{About $2^{32}$ operations and 64 Gigabytes of memory}

At several steps of the algorithm, we are faced with the following problem. We have to find all binary sequences $\left(x_{i}\right), 1 \leq i \leq 64$, such that $\sum_{i=1}^{64} x_{i} a_{i} \equiv b_{1}\left[m_{1}\right]$, where $m_{1} \simeq 2^{32}$, and where $b_{1}$ is a fixed value. We here show in detail how to do this in about $2^{32}$ operations and we estimate the needed size of memory.

Implementation 1 Let $R$ be a file that can contain $2^{32}$ words of 32 bits, and let $S$ be a file that can contain $2^{32}$ words of 64 bits. We store in the file $R$ intermediate results and $S$ will be the file of solutions $\left(x_{i}\right)$.

Step a : For each $\left(x_{1}, \ldots, x_{32}\right)$ we compute $k=b_{1}-\sum_{i=1}^{32} x_{i} a_{i}$ modulo $m_{1}$ and store $\left(x_{1}, \ldots, x_{32}\right)$ in file $R$ at address $k$. If a solution $\left(x_{1}^{\prime}, \ldots, x_{32}^{\prime}\right)$ was already introduced at that address, then $\left(x_{1}, \ldots, x_{32}\right)$ is dropped. In implementation 2 however all intermediate results will be stored. This step a needs about $2^{32}$ operations since there are $2^{32}$ sequences $\left(x_{1}, \ldots, x_{32}\right)$.

Step b : For each $\left(x_{33}, \ldots, x_{64}\right)$ then $k^{\prime} \equiv \sum_{i=33}^{64} x_{i} a_{i}$ modulo $m_{1}$ is computed.

First case: The register at address $k^{\prime}$ in file $R$ contains a sequence $\left(x_{1}, \ldots x_{32}\right)$. Then $\left(x_{1}, \ldots, x_{32}, x_{33}, \ldots x_{64}\right)$ is stored in $S$ because then $k^{\prime} \equiv \sum_{i=33}^{64} x_{i} a_{i}\left[m_{1}\right] \equiv b_{1}-\sum_{i=1}^{32} x_{i} a_{i}\left[m_{1}\right]$, which entails $\sum_{i=1}^{64} x_{i} a_{i} \equiv b_{1}\left[m_{1}\right]$.

Second case: The register at address $k^{\prime}$ in file $R$ is empty. Here the following $\left(x_{33}, \ldots, x_{64}\right)$ is considered. This step $b$ also needs about $2^{32}$ operations.

Hence after $\mathbf{a}$ and $\mathbf{b}$ we will have a solution $\left(x_{i}\right)$ for each value $k^{\prime}$ such that $\sum_{i=1}^{64} x_{i} a_{i} \equiv$ $b_{1}\left[m_{1}\right]$ and $\sum_{i=33}^{64} x_{i} a_{i}=k^{\prime}$

Implementation 2 It is possible to improve implementation 1 in order to obtain all solutions $\left(x_{1}, \ldots, x_{64}\right)$ still in about $2^{32}$ operations and with about $4 \cdot 2^{32} \cdot 32$ bits of memory. Let $X$ be a new file for $2^{32}$ words of 32 bits.

Step a If the register with address $k$ computed from $\left(x_{1}, \ldots, x_{32}\right)$ already contains the intermediate result $\left(x_{1}^{\prime}, \ldots, x_{32}^{\prime}\right)$, then shift $\left(x_{1}^{\prime}, \ldots, x_{32}^{\prime}\right)$ to the address $\left(x_{1}, \ldots, x_{32}\right)$ of $X$, and introduce $\left(x_{1}, \ldots, x_{32}\right)$ in the register with address $k$ of file $R$. 
Step $b$ We only have to consider the first case. Sequence $\left(x_{1}, \ldots, x_{32}\right)$ is found at address $k^{\prime}$ in file $R$. Then $\left(x_{1}^{\prime}, \ldots, x_{32}^{\prime}\right)$ if any is found at address $\left(x_{1}, \ldots, x_{32}\right)$ of $X$. Next $\left(x_{1}^{\prime \prime}, \ldots, x_{32}^{\prime \prime}\right)$ if any is found at address $\left(x_{1}^{\prime}, \ldots, x_{32}^{\prime}\right)$ of $X$ and so on untill an empty register appears. In file $S$ are stored successively $\left(x_{1}, \ldots, x_{32}, x_{33}, \ldots, x_{64}\right),\left(x_{1}^{\prime}, \ldots, x_{32}, x_{33}, \ldots, x_{64}\right)$, $\left(x_{1}^{\prime \prime}, \ldots, x_{32}^{\prime \prime}, x_{33}, \ldots, x_{64}\right) \ldots$ That way all solutions are exhibited and stored in $S$ in about $2.2^{32}$ operations.

Size of memory We need about $4 \cdot 2^{32} .32$ bits $=64$ Gigabytes and 1.4 Gigabytes Disks are available today. The size of memory needed is thus high but not unrealistic.

\subsection{Some steps can be done once for all}

In our algorithm, Steps 2, 3, 4, and 6 do not depend on the value of $b$. The numbers $a_{i}$ being publicly disclosed, computations in those steps can be performed once for all.

\section{Generalizations of our algorithm to other sizes of Knapsacks}

The problem. Let $a_{1}, \ldots, a_{s}$, be fixed integers of $A$ binary digits. If $T$ is a plaintext of $s$ binary symbols, i.e. $T=x_{1} \ldots x_{s}$, then $b=\sum_{i=1}^{s} x_{i} a_{i}$ is the proposed hashed value. The integer $b$ has $B$ binary digits i.e. $B \simeq A+\log _{2} s$.

We have seen in Section 1 that it is possible to find a sequence $\left(x_{i}\right)$ such that $\sum_{i=1}^{s} x_{i} a_{i}=$ $b$, when $b$ is given, in about $2^{32}$ operations, when $s=256$ and $B=128$. We will now consider some other values for $s$ and $B$.

Case $s=512$ and $B=160$. It is still possible to find a sequence $\left(x_{i}\right)$ when $s=512$ and $b=160$ in about $2^{32}$ operations: we will just have to add a stage in our algorithm. Schematically we will then have the following. Notice that $2^{32}$ is the evaluation of the number of solutions.

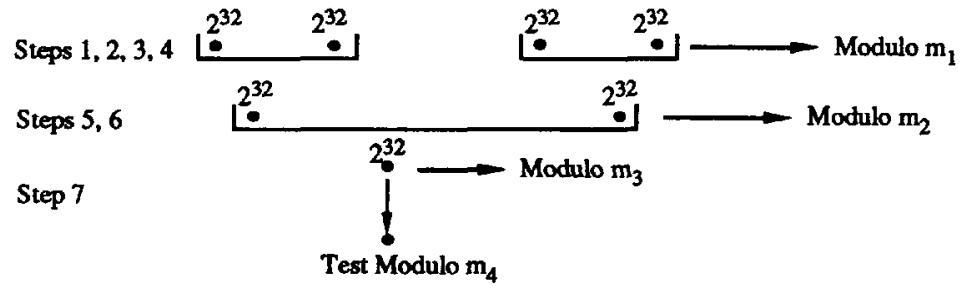

Explanation of the scheme: $m_{1}, m_{2}, m_{3}, m_{4}, m_{5}$ are pairwise coprime integers such that $m_{i} \simeq 2^{32}, i=1,2,3,4$, or 5 , and $m_{1} m_{2} m_{3} m_{4} m_{5}>2^{160}$.

Let $b_{i}$ be the residue of $b$ modulo $m_{i}$. 
- At Stage (1), we find about $2^{32}$ solutions $\left(x_{i}\right)$ such that $\sum_{i=1}^{64} x_{i} a_{i} \equiv b_{1}\left[m_{1}\right]$, about $2^{32}$ solution $\left(x_{i}\right)$ such that $\sum_{i=65}^{128} x_{i} a_{i} \equiv 0\left[m_{1}\right], \ldots$, about $2^{32}$ solutions $\left(x_{i}\right)$ such that $\sum_{i=449}^{512} x_{i} a_{i} \equiv 0\left[m_{1}\right]$

- At Stage (2), we regroup in pairs the solutions found at Stage (1) to find about $2^{32}$ solutions $\left(x_{i}\right)$ such that $\sum_{i=1}^{128} x_{i} a_{i} \equiv b_{2}\left[m_{2}\right], \ldots$, about $2^{32}$ solutions $\left(x_{i}\right)$ such that $\sum_{i=385}^{512} x_{i} a_{i} \equiv 0\left[m_{2}\right]$. Notice that these sequences $\left(x_{i}\right)$ also verify $\sum_{i=1}^{128} x_{i} a_{i} \equiv$ $b_{1}+0\left[m_{1}\right], \ldots$, and $\sum_{i=385}^{512} x_{i} a_{i} \equiv 0+0\left[m_{1}\right]$.

- At Stage (3), we regroup in pairs the solutions of Stage (2) to find about $2^{32}$ solutions $\left(x_{i}\right)$ such that $\sum_{i=1}^{256} x_{i} a_{i} \equiv b_{3}\left[m_{3}\right]$ and $2^{32}$ solutions $\left(x_{i}\right)$ such that $\sum_{i=257}^{512} x_{i} a_{i} \equiv 0\left[m_{3}\right]$. Then at Stage (4) we regroup these solutions to finally find a solution $\left(x_{i}\right)$ at Stage (5) such that $\sum_{i=1}^{512} x_{i} a_{i} \equiv b_{i}\left[m_{i}\right], i=1,2,3,4$ or 5 .

By the chinese remainder theorem, we then found a sequence $\left(x_{i}\right)$ such that $\sum_{i=1}^{512} x_{i} a_{i}=b$, as wanted.

Other Generalizations. With the same technique, by using one, two, etc. more stages, and still with about $2^{32}$ operations, we can solve the cases where $b=192$ and $s=1024$, or $b=224$ and $s=2048$ etc.

So, our algorithm can solve these cases in about $2^{32}$ operations:

\begin{tabular}{c|c} 
Upper bound for \# binary digits $t$ of $b$ & $\begin{array}{c}\text { Lower bound for \# binary symbols } s \\
\text { of plaintext T. }\end{array}$ \\
\hline 96 & 128 \\
128 & 256 \\
160 & 512 \\
192 & 1024 \\
224 & 2048 \\
etc. &
\end{tabular}

If $b=64$ and $s=128$, using the same process, a sequence $\left(x_{i}\right)$ will be found in about $2^{16}$ operations. Schematically: 


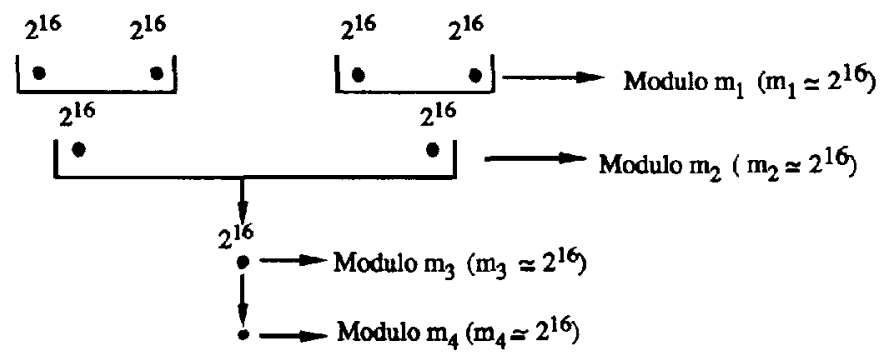

\section{Optimality}

\subsection{A slight improvement}

In section 2.4.1, we have seen that the expected number of collisions obtained is at least 12. If the expected size of $S_{\gamma}$ defined in 2.4 .2 would be $2^{30}$ instead of $2^{32}$, we would have an expected number of at least $2^{16}>3$ collisions which is a satisfactory prospect.

Let $m_{1}=2^{t}, m_{2}=2^{u}, m_{3}=2^{v}$ and $m^{4}=2^{w}$. Setting $t$ to $32, u$ to 31 and $v$ to 32 , then by corollary 1 , the expected size of $S_{\gamma}$ becomes $2^{256-4 t-2 u-v}=2^{30}$.

\subsection{The designed sizes are best possible. Needlessness of an extra stage}

We first point out that if separating step 7 in section 1.1 from the final scanning of $S_{\gamma}$ modulo $m_{4}$ helps assessing the expectations in section 2 , however the result of all computations is the same if we directly compute modulo $m_{3} m_{4}$ in step 4 . Disregarding the refinement above and considering that $2^{v}=m_{3} m_{4}$, we have that at the end of the process the expected number of solutions is $2 r$ with $r=256-4 t-2 u-v$.

The first constraints are thus $t \geq 0, u \geq 0, v \geq 0$ and

$$
\text { (1) } 256-4 t-2 u-v \geq 0
$$

But we need that $m_{1} m_{2} m_{3} m_{4} \geq b$ for relations 1 and 2 of section 1.1 :

$$
t+u+v \geq 128 \text {. }
$$

Now the expected sizes of the sets we have to deal with are

$$
2^{64-t} \text { and } 2^{128-2 t-u} \text {. }
$$

The maximum of these numbers is to be as small as possible and we thus have that

$$
\text { (3) } \quad 64-t=128-2 t-u \text {. }
$$

Adding (1) and (2) we get

$$
128-t-u \geq 2 t,
$$


and since by (3) we have that $t+u=64$, we obtain $0 \leq t \leq 32$. We thus minimize $2^{64-t}$ by setting $t=32$ and thus $u=32, v=64$ which were the selected values. With one more stage, the sizes of the sets to be recorder would be $2^{32-t}, 2^{64-2 t-u}, 2^{128-4 t-2 u-v}$ where $m_{1}=2^{t}, m_{2}=2^{u}, m_{3}=2^{v}, m_{4}=2^{w}$.

We here have the constraints $t+u+v+w \geq 128$ and $256-8 t-4 u-2 v-w \geq 0$, from which $128-4 t-2 u-v \geq 3 t+u$. If we attempt to obtain a smaller size than previously, we need that $3 t+u \leq 128-4 t-2 u-v \leq 32$ and $64-2 t-u \leq 32$, which implies $t \leq 0$. Since $2^{t}=m_{1}$ we must have $t=0$. This means that the considered extra stage is useless.

\section{Conclusion}

The technique is very efficient for a lot of values of $s$ and $t$. With the values sugested by I. Damgård, we can find a sequence $\left(x_{i}\right)$ using a number in the region of $2^{32}$ operations and about 60 Gigabytes of memory. This is high, but not impracticable. Still, if $t=256$ and $s=512$ for example, finding a sequence $\left(x_{i}\right)$ in a number in the region of $2^{32}$ operations remains an open problem.

\section{References}

[1] P. Camion, Can a Fast signature Scheme Without Secret Key be Secure?, in AAECC-2, Lecture Notes in Computer Science $\mathrm{n}^{\circ} 228$, Springer-Verlag.

[2] P. Camion and Ph. Godlewski, Manipulation and Errors, Localization and Detection, Proceedings of EuroCrypt'88, Lecture Notes in Computer Science $n^{\circ} 330$, SpringerVerlag.

[3] M. Campana and M. Girault, How to Use Compressed Encoding Mechanisms in Data Protection, Securicom 88, March 15-17, pp. 91-110.

[4] D. Dacunha-Castelle and D. Revuz, Recueil de problèmes de calcul des probabilités, Masson et Cie, Paris, 1970.

[5] I. Damgård, Design Principles for Hash Functions, Proceedings of Crypto'89, Springer-Verlag.

[6] D.W. Davis and W.L. Price, Security for computer Networks, John Wiley and Sons, Chichester 1984.

[7] M. Girault, Hash Functions Using Modulo-n Operations, Proceedings of EuroCrypt'87, Springer-Verlag.

[8] J.K. Gibson, Some comments on Damgard's hashing principle, Electronic letters 19th July 1990 , Vol. $26 \mathrm{n}^{\circ} 15$. 